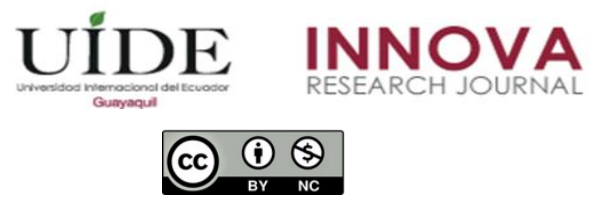

INNOVA Research Journal, ISSN 2477-9024

(Septiembre-Diciembre 2019). Vol. 4, No.3.2 pp. 89-97

DOI: https://doi.org/10.33890/innova.v4.n3.2.2019.1063

URL: http://revistas.uide.edu.ec/index.php/innova/index

Correo: innova@uide.edu.ec

\title{
La Hotelería en Quito: ¿industria competitiva?
}

\section{The Hospitality in Quito: competitive industry?}

María Dolores Quintana Lombeida

Universidad UTE, Ecuador

Autor para correspondencia: maria.quintana@ute.edu.ec

Fecha de recepción: 12 de julio de 2019 - Fecha de aceptación: 18 de noviembre de 2019

\section{Resumen}

Quito como capital del Ecuador es parte de esta investigación a través de un estudio descriptivo con técnicas de observación y revisión bibliográfica en los emprendimientos locales y grandes empresas. Entre los elementos hallados se destaca que desde la década de los ochentas las franquicias y cadenas hoteleras internacionales rompieron el mercado con calidad y un alto desempeño que estimularon los porcentajes de ocupación corporativa. Adicionalmente el Ecuador posee un nuevo Reglamento de Alojamiento Turístico, vigente desde el 2015, donde ya se incorporan las categorías de: haciendas turísticas, lodges con la potencialización de infraestructuras patrimoniales y resorts con todos los servicios de alimentos, bebidas y lúdicos. Entre los resultados hallados se destaca que una de las mayores ventajas competitivas son las tarifas de hospedaje reconocidas como una de las más económicas de América. Además, el aporte que refleja la industria de alojamiento y servicios de comida ocupa el $27.7 \%$ de los ingresos por generación de empleo del sector y que la mayor concentración de empleabilidad está en la Zona Especial Turística "La Mariscal”. Finalmente se destaca la limitación en capacidades de inversión en los establecimientos pequeños en tecnología e innovación frente a las grandes franquicias y cadenas.

Palabras clave: hotelería; Quito; franquicias; emprendimientos

\begin{abstract}
Quito as the capital of Ecuador is part of this research through a descriptive study with observation techniques and bibliographic review in local enterprises and large companies. Among the elements found is that since the eighties international franchises and hotel chains broke the market with quality and high performance that defied the percentages of corporate activity. In addition, Ecuador has a new Tourism Accommodation Regulation, in force since 2015, where the categories of: tourist farms, lodges with the potentialization of heritage infrastructure and resorts with all food, beverage and recreational services are already incorporated. Among the results found, one of the greatest competitive advantages is the lodging rates recognized as one of the cheapest in America. In addition, the report that reflects the accommodation and food service industry occupied $27.7 \%$ of the income generated by employment generation in the sector and the highest concentration of employability is in the Special Tourist Zone "La Mariscal". Finally, the limitation in investment capacities in the small ones in technology and innovation in front of the big franchises and chains stands out.
\end{abstract}


Key words: hospitality; Quito; franchises; entrepreneurship

\section{Introducción}

Quito es considerada como una de las ciudades más antiguas de América, se ha potencializado como un destino turístico por sus riquezas patrimoniales y recursos, que han sido un atractivo para diferentes nacionalidades. Su sinergia en un pequeño territorio abarca una amplia historia.

Quito ya existía antes de la fundación española del 6 de diciembre de 1534. En la época aborigen, centro del antiguo Reino de los Quitus y formó parte del imperio Inca, tras la invasión del Huayna Cápac quien, a su muerte, entrego esta ciudad a su hijo Atahualpa.

Cuando los españoles llegaron a estos territorios, tras la caída del Imperio Inca, Diego de Almagro emprendió la fundación de la ciudad de Santiago de Quito cerca de la actual Riobamba. Meses después, Sebastián de Benalcázar tomó posesión definitiva de la ciudad aborigen de Quito y fundó, oficialmente, la ciudad de San Francisco de Quito el 6 de diciembre de 1534, sobre los escombros de la antigua ciudad abandonada por Rumiñahui (Zambrano, 2005, pág. 5).

Fue fundada sobre una antigua ciudad Inca y la UNESCO la declaró en 1978, como Patrimonio de la Humanidad, los territorios declarados como patrimonios de la humanidad tienen la obligatoriedad de ser preservados por el hombre como parte de su memoria. El centro histórico de la capital ecuatoriana es el mejor salvaguardado y menos alterado de toda América, cuenta con suntuosas iglesias, monasterios, museos y una riqueza que es atractiva para turistas durante todo el año.

Según el Instituto Nacional de Estadísticas y Censos (INEC) el crecimiento poblacional es acelerado y ocupará la mayor población en el Ecuador para el año 2020, se prevé que alcance 2`781.641 habitantes, la geografía por ser prolongada y estrecha se ha expandido hacia los valles.

La ciudad de Quito está situada en el valle de Quito en la vertiente interandina de la Cordillera Occidental, alineada de sur a norte por más de $50 \mathrm{~km}$, flanqueada por el volcán Pichincha a $4680 \mathrm{msnm}$. Y el Atacazo por el oeste, y por el sistema de

PuengasíGuanguiltagua al este. Se asienta sobre una llanura lacustre que ocupa el eje de la depresión a una cota promedio de $2800 \mathrm{msnm}$, y que corresponde a una planicie relacionada con una falla geológica longitudinal de 45 km. (Rivadeneira \& León, 2017)

La ciudad es un territorio con alto riesgo sísmico, proclive a condiciones de altas precipitaciones y tendencia a erupciones volcánicas debido a su ubicación.

En el contexto económico la ciudad se ha potencializado en la generación de empleo, en las divisiones que se prioriza la movilización económica están: el sector de la construcción con el $53.7 \%$, las actividades profesionales científicas y técnicas en el $49.7 \%$, actividades de órganos extraterritoriales con $49.3 \%$, en noveno puesto están el alojamiento y servicios de comida con el $27.7 \%$ (El telegrafo, 2016). 
De tal manera el sector de alojamiento y servicios es radical para el desarrollo económico de Quito, su infraestructura patrimonial y moderna facilita el progreso de una industria hotelera, esto en suma a los recursos naturales que posee, facilita las posibilidades de visita de turistas.

La industria hotelera en la ciudad de Quito posee un crecimiento radical desde los años ochenta, antes de ello solo existían emprendimientos locales y algunas marcas extranjeras como Hilton y Oro verde, allí se marca un punto radical donde ingresan franquicias y cadenas a una ciudad representada como Patrimonio de la Humanidad y con recursos históricos y naturales atractivos. Por lo cual el objetivo de la investigación es determinar la competitividad del hotelería en Quito para establecer la realidad del sector. Dicho objetivo se canalizará a través de la revisión de tres objetivos específicos el primero, identificar la afluencia turística actual de la ciudad de Quito que permita determinar los principales nichos de mercado. El segundo objetivo se refiere a Evaluar el Reglamento vigente de establecimientos turísticos afín de detectar su impacto en la categorización propuesta y el tercer objetivo específico diagnosticar las condiciones de las franquicias y cadenas en Quito para detectarlas como factor clave de impacto positivo o negativo en la industria.

Se reconocen a dos zonas especiales turísticas, la primera es la Zona de La Mariscal y la segunda es la Zona del Centro Histórico de Quito; La Mariscal aporta con el 24\% del total de empleos en el sector del turismo de todo el Distrito Metropolitano de Quito DMQ; debido a su alto tráfico de turistas, la alta densidad de motores de atracción y la cercanía a los sitios más visitados en la franja urbana.

Conforme al (Sistema Institucional de Indicadores Turísticos SIIT, 2019) el 85\% de los hoteles en todo el Ecuador son pequeñas y medianas empresas que son propiedad en su mayoría de ecuatorianos; quienes no han desarrollado alta inversión en tecnología, calidad y mejoras en servicios al nivel de las marcas internacionales, las franquicias y cadenas hoteleras revierten y generan una mayor calidad e inversión en calidad y lujo.

Franquicias y cadenas hoteleras

\section{Materiales y Métodos}

El estudio que se realizo fue distribuido en conformidad de los tres objetivos específicos en el estudio de la afluencia turística de Quito se investigó en las bases de datos provistas por la empresa municipal Quito Turismo y a través del Sistema Institucional de Indicadores Turísticos SIIT con referencia la análisis del Reglamento de Establecimientos turísticos se desarrolló una revisión bibliográfica y de la literatura del reglamento finalmente en referencia al análisis de franquicias y cadenas hoteleras se hizo una revisión bibliográfica en textos, artículos científicos al respecto de antecedentes históricos de la industria hotelera en Quito y la posteridad con el crecimiento de los negocios en ese modelo, se desarrolló un estudio descriptivo de datos etimológicos y de crecimiento del sector. 


\section{Resultados}

Entre los resultados hallados se destacan tres aristas: la primera con relación a Quito y la afluencia turística donde se menciona los desplazamientos de turistas y principales destinos de visita, la segunda se refiere a la categoría y clasificación de establecimientos conforme al nuevo Reglamento de Alojamiento del 2015, un comparativo de las tarifas a nivel de otras ciudades de Latinoamérica y la tercera arista comprende la influencia de Franquicias y cadenas hoteleras en Quito:

\section{Quito, afluencia turística}

La demanda turística abarca tanto el desplazamiento necesario como todos los bienes y servicios requeridos por el consumidor durante dicho desplazamiento (Iranzo, 2012, pág. 60). Quito como capital del Ecuador, cuenta con una gran afluencia de viajeros en los últimos tres años, posee un crecimiento entre el $4 \%$ y el $6.1 \%$ desde el 2017 al 2018. Se destacan a los sitios más visitados: Mitad del Mundo 86\%, Centro Histórico 67\%, Teleférico 12\%, La Mariscal 12\%, El Panecillo 11\%, La Ronda 9\% e Iglesias con un $11 \%$.

Tabla N. 1

Datos Turísticos de Quito

\begin{tabular}{llllll}
\hline \multicolumn{1}{c}{ Turistas del DMQ } & $\mathbf{2 0 1 6}$ & $\mathbf{2 0 1 7}$ & $\mathbf{2 0 1 8}$ & $\mathbf{2 0 1 9}$ & Unidad \\
\hline $\begin{array}{l}\text { Llegada de turistas a Quito } \\
\text { Tasa de crecimiento }\end{array}$ & 627.626 & 652.931 & 692.492 & 709.984 & Turistas \\
& $-12 \%$ & $4 \%$ & $6.1 \%$ & $2.5 \%$ & $\begin{array}{l}\text { Respecto año } \\
\text { anterior }\end{array}$ \\
$\begin{array}{l}\text { Proyección al 2021 (número de } \\
\text { turistas) }\end{array}$ & & 689.673 & & Turistas \\
\hline
\end{tabular}

Recuperado de: https://www.quito-turismo.gob.ec/estadisticas/llegadas-y-salidas-quito.

Los nichos de mercado son lugares específicos o particulares (...) nuevos mercados en los que existen necesidades latentes que, no atendidas, ya sea porque se desconoce su existencia de su potencial o porque no han interesado las empresas que usualmente operan grandes segmentos de mercado (Melgar, 2000).

Los datos reflejan que los periodos de "la estadía promedio que permanece un turista extranjero en Quito fue de cinco a seis días en 2016; mientras que la estancia media hotelera fue de 1.56 noches" (Sistema Institucional de Indicadores Turisticos, 2017) lo cual pone en manifiesto que el turista extranjero no permanece en la capital ecuatoriana, sino que lo considera como un destino de paso, por lo cual su consumo no es sustancial; esto obliga a los establecimientos a desplegar estrategias comerciales que logren un atractivo frente a la oferta de otros destinos internacionales.

Es relevante mencionar que el crecimiento de la llegada de turistas a Quito fue positivo entre el año 2017- 2018 y persiste su progreso en el año 2019. Los principales países demandantes del turismo en Quito son: Estados Unidos, Colombia, Ecuador, España, Canadá, Alemania, Argentina, Perú, Reino Unido, Francia, México, Chile, Brasil e Italia. Tras un descenso en el año 2016 del -12\%. La proyección al año 2021 es de 689.673 turistas. 


\section{Categoría y clasificación de establecimientos}

Los establecimientos de alojamiento brindan hospeda por un tiempo determinado en retribución de unidades monetarias, en este hospedaje se pueden ofertar servicios adicionales, lo que determinara su clasificación y categoría (Rodriguez \& otros, 2012, pág. 98).

En el Ecuador el establecimiento de alojamiento oferta servicios de alojamiento y alimentación adoptando una clasificación y categorías. Según el Reglamento de Alojamiento Turístico vigente desde el año 2015. La industria hotelera en el país se clasifica en: hotel ( 2 a 5 estrellas), hostal ( 1 a 3 estrellas), hostería ( 3 a 5 estrellas), hacienda turística ( 3 a 5 estrellas), lodge (3 a 5 estrellas), resort (4 a 5 estrellas), refugio (categoría única), campamento turístico (categoría única) y casa de huéspedes (categoría única).

Las categorías de los establecimientos de alojamiento fueron actualizadas en el reglamento vigente, lo cual facilita la promoción turística internacional, ya que su equivalencia corresponde a nivel internacional; en contraste a la anterior categoría que planteaba a las de: lujo, primera y segunda categoría indistintamente. A nivel de Latinoamérica, los hoteles de cinco estrellas tienen tarifas muy competitivas, al respecto, en un cotejo entre: Lima, Quito, Bogotá, Buenos Aires y Brasilia; se destaca que la tarifa de Quito es más baja que en Bogotá, que se antepone, debido a sus altos niveles de inseguridad, como lo describe la Tabla N 2. Colombia es el país más peligroso, según el análisis del sector turístico en ciento treinta y seis naciones del mundo, el tópico se orienta al terrorismo y violencia que se desata en este territorio.

"Los cinco países más peligrosos son Nigeria, Pakistán, El Salvador, Yemen y Colombia” (Panam Post, 2017).

Por la cercanía de Ecuador con Colombia y al ser un factor exógeno e incontrolable contrarresta la propuesta de tarifas frente a la región.

Tabla N. 2

Tarifas en hoteles 5 estrellas de países en Latinoamérica

\begin{tabular}{lll}
\hline País & Categoría & Tarifa Rack \\
\hline Lima & 5 estrellas & $\$ 449$ \\
Quito & 5 estrellas & $\$ 174$ \\
Bogotá & 5 estrellas & $\$ 191$ \\
Buenos Aires & 5 estrellas & $\$ 339$ \\
Brasilia & 5 estrellas & $\$ 103$ \\
\hline \multicolumn{3}{c}{ Adaptado de: expedia.com }
\end{tabular}

Los servicios de los establecimientos cinco estrellas en el Ecuador no difieren en gran manera de su competencia en Latinoamérica con respecto a los servicios lúdicos, la infraestructura y el área de habitaciones.

El Reglamento en Ecuador no tiene exigencias detalladas como los de otros países de la región, en el actual inclusive se permiten la creación de hoteles de 5 estrellas con tan solo 5 habitaciones, no se detalla exhaustivamente las prescripciones para cada una de las categorías y clasificaciones. 
A nivel mundial la hotelería se desarrolla por el modelo de internacionalización de marcas a través de las franquicias, su renta, fusión, contratación con locales adquiridos o de posesión de otros, la expansión fue bajo distintas formas pero surgió un adelanto industrial. "No se sabe con exactitud en dónde se originó el franquiciamiento, pero sí se reconoce que es en Estados Unidos donde ha tenido un mayor desarrollo" (Asociación Internacional de Franquicias, 2012) en el sector del alojamiento existen muchos giros de negocio que provienen de Estados Unidos y que han expandido su marca.

El siglo XIX y XX estuvieron caracterizados por la hotelería europea, la segunda mitad de este último siglo lo fue de la hotelería norteamericana y sus formas, que han modelado de manera clara tanto la hotelería como el turismo de la época moderna (Jimenez, 2008)

El modelo de franquicia nace con la influencia de recursos que posee la ciudad y que podrían ser visitados. La industria hotelera reconoce tres etapas: la primera etapa está en los años cincuenta con la construcción y compra de cadenas hoteleras en el mundo, en la segunda etapa surgen desde los años ochenta, donde crecen aceleradamente la expansión de las franquicias con contrataciones internacionales de administración y arriendo, finalmente, la tercera etapa en los años noventa donde se contrata, arrienda, fusiona y administra hoteles propios y de terceros (Pérez, Puig, \& Fernández, julio - diciembre 2016).

En Quito la inversión internacional se hizo presente aproximadamente desde los años ochenta, con la entrada de franquicias y cadenas internacionales, que se consolidaron como altamente competitivas y dirigida a nichos de mercado de mayor nivel. Como lo menciona la Tabla N. 3 las franquicias y cadenas cuentan con una gran cantidad de habitaciones con respecto a hoteles de emprendimientos locales, además de servicios e infraestructura conforme a la normativa del país, que procede la franquicia y cadena. De esta forma su exigencia en servicios, formación de personal, instalaciones, procesos, y operaciones son mucho más exigentes que las que solicita el Reglamento de Alojamiento.

Tabla N. 3

Franquicias y cadenas en la ciudad de Quito

\begin{tabular}{ll}
\hline Nombre Establecimiento & Número de habitaciones \\
\hline Best Western Plaza Hotel & 110 \\
Hotel Dann Carlton Quito & 210 \\
Hotel NH Collection Quito Royal & 112 \\
Hotel Sheraton Quito & 138 \\
Hotel Wyndham Quito Airport & 150 \\
EB Hotel By Eurobuilding Quito & 95 \\
Hotel Le Parc & 30 \\
Wyndham Garden Hotel Quito & 90 \\
Hilton Colón Quito & 255 \\
Hotel JW Marriott Quito & 257 \\
Hotel Quito & 215 \\
Hotel Swissotel Quito & 383 \\
Akros Hotel By BlueBay & 128 \\
Hotel Holiday Inn Express & 141 \\
Hotel Park Inn by Radisson & 55 \\
Hotel Ibis Quito & 151 \\
\end{tabular}

Fuente: (Federación Hotelera del Ecuador, 2019) (AHOTEC) 
Se resaltan las 10 cadenas hoteleras más grandes que operan en el mundo, esta información la proporciona la revista Hotels en un ranking propuesto en el 2017, conforme la Tabla N. 4. Se destaca a la cadena Marriot International como la más grande con 5952 hoteles.

Tabla N. 4

Cadenas de hoteles más grandes del mundo

\begin{tabular}{lc}
\hline \multicolumn{1}{c}{ Cadena } & Número de hoteles \\
\hline Marriot International & 5952 \\
Hilton & 4875 \\
InterContinental Hotels & 5174 \\
Group (IHG) & \\
Wyndham Hotels Group & 8000 \\
Shanghai Jin Jiang & $>6000$ \\
International Hotel & \\
Group Inc. & \\
AccorHotels & \\
Choice Hotels & 4144 \\
International Inc & 6500 \\
BTG Hommeins Hotels & \\
Group & 3402 \\
$\quad$ China Lodging Group & 3269 \\
$\quad$ Best Western Hotels \& & 3677 \\
$\quad$ Resorts & \\
\hline \multicolumn{2}{c}{ Adaptado de: (El economista, 2017) }
\end{tabular}

Entre las empresas más representativas en el mundo, la presencia que destaca en Quito Son:

\section{Tabla N.5}

Cadenas que operan en Quito con mayor representación internacional

\begin{tabular}{lc}
\hline \multicolumn{1}{c}{ Cadena } & Hoteles en Quito \\
\hline Marriot International & (JW Marriot Hotel Quito), \\
Hilton & (Hilton Colon Quito), \\
InterContinental Hotels Group & (Holiday inn Express) \\
Wyndham Hotels & (Wyndham Garden Quito hotel, Wyndham Quito Airport hotel), \\
AccorHotels & (Mercure Alameda Quito, Swissôtel Quito, ibis Quito) \\
Choice Hotels International Inc & (San José de Puembo) \\
Best Western Hotels \& Resorts & (Best Western CPlaza Hotel, Best Western Hotel Zen) \\
\hline & Elaborado por: autora
\end{tabular}

Los negocios de empresarios locales, en su generalidad, operan bajo las normativas locales; por lo cual los productos, servicios y exigencias al personal son menores y para contrarrestar esta deficiencia en calidad, muchos de ellos se han esforzado en alcanzar certificaciones como TourCert, Rain Forest Alliance, Buenas Prácticas de Manufactura e ISO, que regularizan y exigen en distintos tópicos como: infraestructura, personal, responsabilidad ambiental, responsabilidad social y por lo tanto los productos y servicios que ofertan. 


\section{Conclusiones}

Quito es una ciudad con gran expansión económica, sin embargo su desarrollo turístico y particularmente en la industria de alojamiento y comestibles y bebidas apenas ocupa el noveno lugar, por lo cual incita la innovación de estrategias que aporten más competitividad y productividad, el ingreso de turistas crece aproximadamente un $2 \%$ cada año lo cual implica un crecimiento lento con resto a otras realidades en América, un elemento crítico en la inseguridad por su cercanía a Colombia, existen mercados ya delimitados internacionalmente sin embargo la negociación con agencias de viajes internacionales y canales de distribución no ha sido efectivo para un crecimiento más representativo por lo cual es necesario incentivar medidas que fomenten la afluencia turística y permanencia en el territorio por más de 1 día.

Los Reglamentos en Ecuador presentan facilidad para la interpretación, en el caso del Reglamento de actividades turísticas debería ser más estricto fomentar a la calidad en los establecimientos locales, Quito presenta tarifas atractivas, pero no tienen alta rentabilidad en una economía con altos costos de operación y poca inversión en pequeñas y medianas empresas del sector.

La influencia de cadenas y franquicias hoteleras se postula en la ciudad con la presencia de marcas representativas a nivel mundial, esto hace que los emprendimientos locales tengan menores posibilidades de desarrollo ya que deben invertir, importar tecnología y operar con mano de obra costosa, mientras que el branding para las grandes empresas facilita el acceso al consumidor.

\section{Bibliografía}

Asociación Internacional de Franquicias. (2012).

El economista. (21 de agosto de 2017). América Economia. Obtenido de Estas son las 10 cadenas hoteleras más grandes del mundo: https://lifestyle.americaeconomia.com/articulos/estasson-las-10-cadenas-hoteleras-mas-grandes-del-mundo

El telegrafo. (06 de diciembre de 2016). Quito, puntal económico de Ecuador. La ciudad cumple 482 años con dos desafíos pendientes.

Federación Hotelera del Ecuador. (09 de Julio de 2019). Guía de Hoteles. Obtenido de https://www.hotelesecuador.com.ec

Iranzo, J. E. (2012). La demanda de turismo. revista de Economía Mundial, 127-149.

Jimenez, A. d. (2008). Las cadenas hoteleras en el mundo y evolución de su operación en México al inicio del siglo XXI . Innovar.

Melgar, J. (2000). El nichologo y los nichos de mercado como nuevos elementos de mercadeo en el siglo XXI. Theorethikos, año III, número 3, julio-septiembre.

Panam Post. (20 de Junio de 2017). Colombia es el país más peligroso para turistas según Foro Económico Mundial. Panam Post.

Pérez, G. D., Puig, M. Z., \& Fernández, R. M. (julio - diciembre 2016). La expansión internacional de la industria hotelera de los países desarrollados como opción estratégica para los países subdesarrollados. Economía y Desarrollo, vol 157 N.2.

Rivadeneira, E. V., \& León, N. M. (2017). Precipitaciones extremas en la ciudad de Quito, provincia de Pichincha- Ecuador . Ingeniería Hidráulica y Ambiental, Recuperado en 13

Esta obra se comparte bajo la licencia Creative Common Atribución-No Comercial 4.0 International (CC BY-NC 4.0) Revista de la Universidad Internacional del Ecuador. URL: https://www.uide.edu.ec/ 
de mayo de 2019, de http://scielo.sld.cu/scielo.php?script=sci_arttext\&pid=S168003382017000200008\&lng=es\&tlng=es. .

Rodriguez, J. M., \& otros. (2012). Influencia de los principios organizativos, parámetros de diseño y factores de contingencia en las estructuras organizativas de las cadenas hoteleras españolas y mexicanas. España: vision libros.

Sistema Institucional de Indicadores Turisticos. (2017).

Sistema Institucional de Indicadores Turísticos SIIT. (25 de Abril de 2019). Datos de EcuadorQuito.

Zambrano, R. (2005). Quito: Patrimonio de la Humanidad. Quito: Libresa. 